\title{
Length of left main coronary artery
}

\author{
Charles Fox, ${ }^{1}$ M. J. Davies, and M. M. Webb-Peploe \\ From the Cardiac Department, St. Thomas' Hospital, London S.E.I; \\ and the Pathology Department, St. George's Hospital, London S.W.I 7
}

The length of the left main coronary artery has been measured in 100 coronary cineangiograms and in I00 postmortem hearts. The results indicate that an early bifurcation of the left main coronary artery is very much more common than previously suspected. The risks of aortic valve operation in cases with an early bifurcation are discussed.

One of the advances in aortic valve surgery has been perfusion of the coronary arteries by direct cannulation during valve replacement. It has been suggested (Furlong et al., 1972) that a short left main coronary artery or a wide angle between its two main branches may result in underperfusion of the left circumflex artery or less commonly the left anterior descending artery. In such cases with an early bifurcation of the left main coronary artery the coronary cannula may selectively perfuse one main branch and to a greater or lesser degree occlude the other. Thus, a portion of myocardium remains underperfused throughout the bypass and infarction may result. The incidence of short left main coronary artery was therefore determined (a) in 100 coronary cineangiograms and (b) in 100 postmortem specimens with normal hearts.

\section{Methods}

The Ioo coronary cineangiograms were carried out in the cardiac department at St. Thomas' Hospital, using Judkins or Sones techniques. The orifice of the main coronary artery could usually be visualized by spill-back of contrast medium during selective injection. The distance between this point and the bifurcation of the left main coronary artery was measured in a single frame of the left selective coronary cineangiogram in the right anterior oblique position. Errors of magnification were corrected by photographing a perforated metal ruler ( $\mathrm{I} \mathrm{cm}$ perforations) at midchest level and adjusting the coronary artery measurement to this standard. In 6 cases, measurement of the left main coronary artery was technically impossible either because the vessel overlay some radio-opaque structure in this projection, or else because of very severe aortic regurgitation which

Received 5 March 1973.

${ }^{1}$ Present address: Department of Medicine, Pahlavi University, Shiraz, Iran. caused the spill-back to be rapidly diluted with nonopaque blood. In one case the circumflex artery arose from the right coronary artery. Thus a total of 107 consecutive cases was examined.

The postmortem hearts of normal size were obtained from Ioo consecutive necropsies performed at St. George's Hospital (by M.J.D.) on cases of accidental death. The hearts were removed from the chest and fixed in formal saline. The length of the left main coronary artery ( $x$ ) was measured as shown in Fig. $\mathbf{I}$.

The volume of the fixed ventricle was then assessed by filling the cavity with glass beads and measuring the displacement of the beads. No volume exceeded $200 \mathrm{ml}$. The left ventricular wall including the interventricular septum was then dissected from the rest of the heart and weighed. All fell within the normal range (120-200 g).

\section{Results}

The lengths of the left main coronary arteries measured in the coronary cineangiographs are shown in

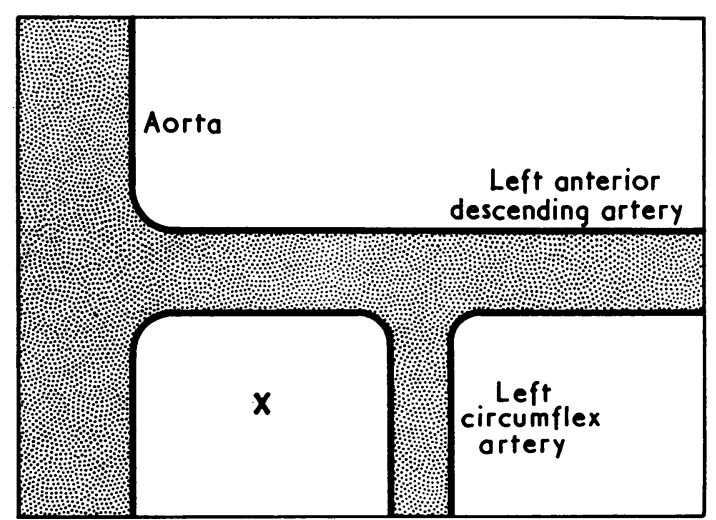

FIG. I Diagram illustrating the measurements of the left main coronary artery at necropsy. 


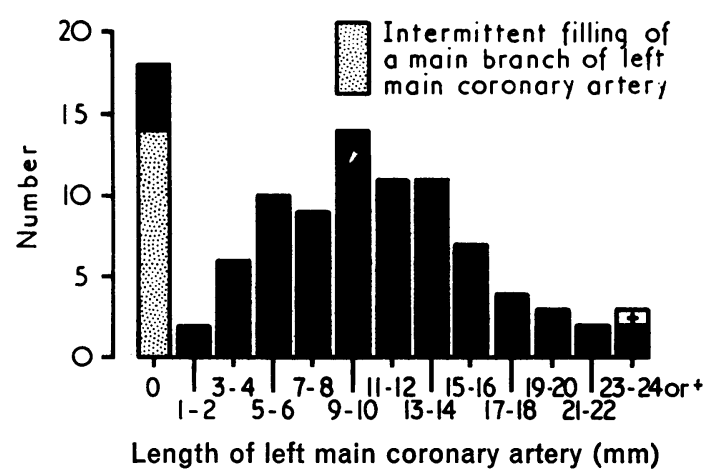

FIG. 2 Length of left main coronary artery in 100 coronary angiograms.

Fig. 2. There is immediate bifurcation in 18 per cent. In 14 per cent intermittent filling of one of the main branches was noted during the infusion of contrast medium. The left circumflex artery filled intermittently in ro per cent and left anterior descending in 4 per cent. This was thought to provide further angiographic evidence of an immediate bifurcation. In 36 per cent the left main coronary artery was $6 \mathrm{~mm}$ or less. Only 5 per cent had left main coronary arteries greater than $20 \mathrm{~mm}$, the longest being $32 \mathrm{~mm}$. The mean length in this series is $9.5 \mathrm{~mm}$.

The lengths of the left main coronary artery in the postmortem group are shown in Fig. 3. Ten per

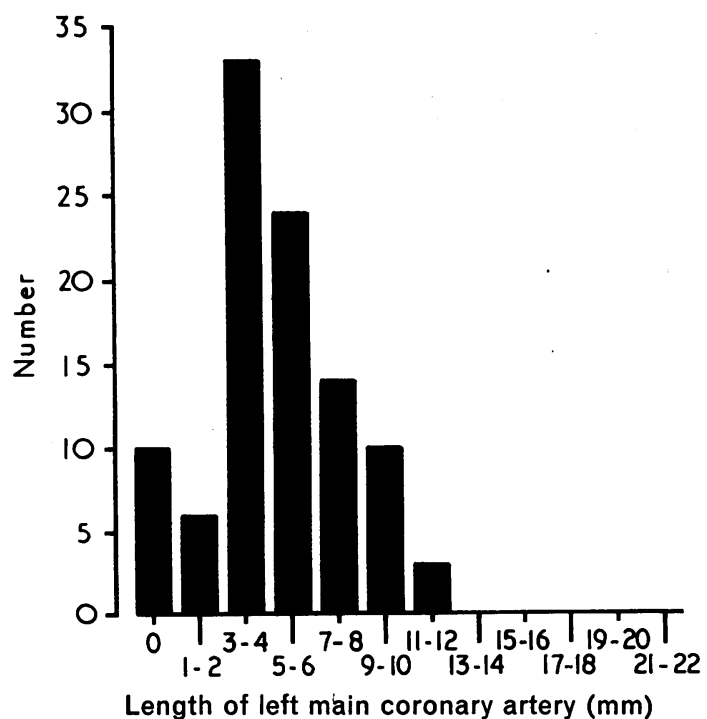

FIG. 3 Length of left main coronary artery in 100 consecutive cadavers with normal left ventricle volume and weight. cent show an immediate bifurcation, and in 73 per cent the length of the left main coronary is $6 \mathrm{~mm}$ or less. In only 3 per cent does the length exceed Io $\mathrm{mm}$. The mean length of the left main coronary artery in these normal-sized postmortem hearts is $5.5 \mathrm{~mm}$.

\section{Discussion}

The only previous series in which the length of the left main coronary artery is tabulated is that of Green, Bernstein, and Reppert (1967). They measured the distance from the ostium to the bifurcation of the left main coronary artery in 50 consecutive necropsy specimens. They found the distance

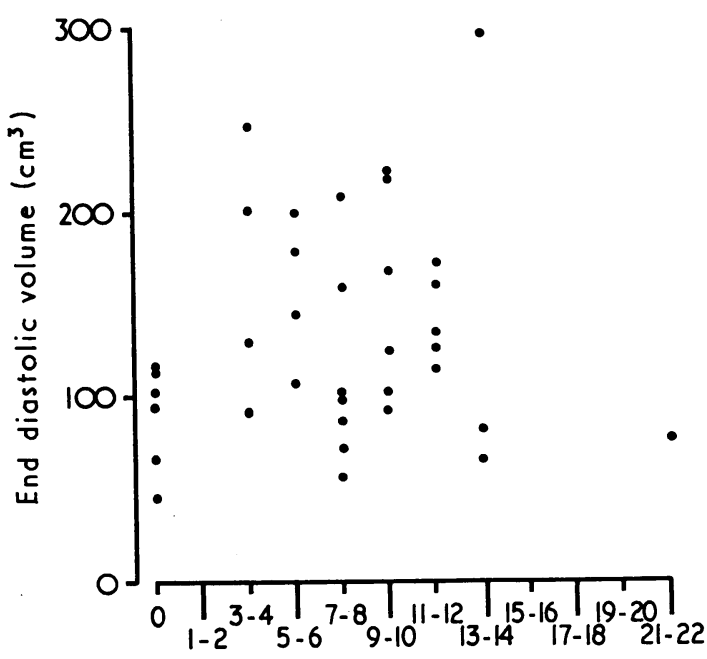

Length of left main coronary artery $(\mathrm{mm})$

FIG. 4 Comparison between end-diastolic volume and left main coronary artery length in 35 cases.

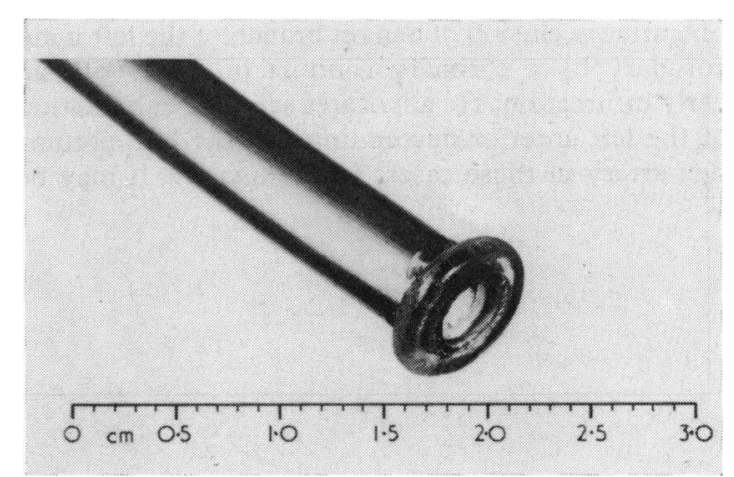

FIG. 5 Portex coronary perfusion catheter in current use. 
greater than $10 \mathrm{~mm}$ in 52 per cent and $6 \mathrm{~mm}$ or less in 26 per cent; thus in general, their measurements are greater than the present findings. The two necropsy series are not comparable as all the hearts in our postmortem group were normal in size, while Green studied a number of enlarged hearts (range of total heart weight 240-500 g). However, Green found no correlation between length of left main coronary artery and the weight of the heart.

In the present angiographic series, a large number of hearts were enlarged, and yet our findings again show a much shorter length of the left main coronary than those of Green. A possible explanation is that the length of the left main coronary artery may be underestimated by rotation, so that the right anterior oblique projection is not at right angles to the plane of the vessel. There is also the limitation of measuring a three-dimensional structure in a single plane. On the other hand, there may be a tendency to overestimate the left main coronary artery on angiography if the two main branches overlie one another so that the bifurcation is obscured. The possibilities of overestimating the length of the left main coronary artery on cineangiography mentioned above may explain the discrepancy between the two groups we now present. Alternatively, the fixative may cause some shrinkage of the vessel at necropsy. The difference in the incidence of immediate bifurcation in these two groups is not statistically significant $(P$ $=0 \cdot I$ ).

We attempted to correlate the length of the left main coronary artery with left ventricular volume. In 37 cases where the left main coronary artery was measured, the left ventricular volume was derived from single-plane left ventricular angiography using the area-length method (Greene et al., 1967). No correlation could be found between these 2 variables (see Fig. 4). Least squares analysis gave an $r$ value of 0.06 .

Green et al. (1967) raise the possibility of preoperative occlusion of a main branch of the left main coronary by a coronary cannula in cases with an early bifurcation. He advocates separate cannulation of the left anterior descending and the left circumflex artery in these cases. Unfortunately, it may be impossible to recognize this situation at the time of operation. Furlong et al. (1972) enlarge on the problem by describing 2 cases of death from preoperative infarction in which the length of the left main coronary artery was $6 \mathrm{~mm}$. In both cases infarction appeared to have resulted from obstruction of one of the main branches of the left main coronary artery by a balloon-tipped perfusion cannula.

Spencer and Malette (1968) discuss the question of early bifurcation of the left main coronary artery. In 82 patients having aortic valve replacement they recognized this condition in 6 , but in one case only at necropsy after the patient had died of a large infarction of the area supplied by the left anterior descending artery. Spencer and Malette quote the findings of Green et al. (1967) and state that an early bifurcation occurs in 5-10 per cent.

The results of our findings, both angiographic and at necropsy, suggest that the incidence of a short left main coronary artery is very much higher than previously reported. Thus all but the shortest coronary cannulae may mechanically obstruct a main branch of the left main coronary artery in a large percentage of cases.

The present practice of the cardiothoracic unit at St. Thomas' Hospital is to perform routine selective coronary angiograms on all patients coming to aortic valve surgery. At operation a Portex coronary perfusion catheter (see Fig. 5) is held in the ostium by a purse-string suture. This minimizes the risk of selective perfusion of a single main branch.

\section{References}

Furlong, M. B., Gardner, T. J., Gott, V. L., and Hutchins, G. M. (1972). Myocardial infarction complicating coronary perfusion during open-heart surgery. Fournal of Thoracic and Cardiovascular Surgery, 63, 185.

Green, G. E., Bernstein, S., and Reppert, E. H. (1967). The length of the left main coronary artery. Surgery, 62, 1021.

Greene, D. G., Carlisle, R., Grant, C., and Bunnell, I. L. (1967). Estimation of left ventricular volume by one-plane cineangiography. Circulation, 35, 6r.

Spencer, F. C., and Malette, W. (I968). Technical considerations of coronary perfusion during aortic valve replacement. Fournal of Cardiovascular Surgery, 9, 562.

Requests for reprints to Dr. M. M. Webb-Peploe, Cardiac Department, St. Thomas' Hospital, London S.E.I. 